\title{
Demand side management of electric vehicle car parks to increase integrated solar power capacity within an existing radial distribution network
}

\author{
M. Kiaee*, A.J. Cruden, S.M. Sharkh \\ Energy Technology Research Group, Engineering Sciences, University of Southampton, Highfield campus \\ Southampton, SO17 1BJ, UK, *Email: m.kiaee@ soton.ac.uk
}

Keywords: Solar power, Electric vehicles, Distribution network, Demand side management, Car parks.

\begin{abstract}
The amount of integrated solar power generation capacity within the UK distribution networks is expected to continue to increase within the next few years. However, the grid has a fixed power transfer capacity, and to avoid the extra cost of power system upgrade or curtailment of renewable power, it would be necessary to store the surplus power from such nondispatchable renewable resources using appropriate energy storage technologies. In this work, a novel control strategy based on extended optimal power flow is introduced to control the charging of electric vehicles located at car parks to increase the solar power generation capacity within a radial distribution network while satisfying the power system constraints and electric vehicle user requirements.
\end{abstract}

\section{Introduction}

The number of electric vehicles (EVs) in the UK is increasing to satisfy carbon emission targets in the transport sector. On average, EVs are parked a significant amount of time during a typical day and usually have an energy storage capacity more than the regular requirement of their users, so there exists the possibility to utilise them as responsive loads to improve the performance and efficiency of the power system.

Transmission networks in the UK are already operating close to their capacity constraints, and adding renewable power generators at transmission level would require upgrading these networks with significant investment, hence connecting generation to distribution networks has become popular. Current distribution networks have been designed on a 'fit and forget' basis, so some technical issues could arise as a result of adding more distributed renewable generation within the network. Such issues include voltage rises due to the connection of generators or reverse power flows, which could result in the violation of network constraints. Therefore, there is a need to make distribution networks active by inclusion of responsive distributed generators (DGs) or controllable demands [1].
Intermittent renewable resources such as solar farms only generate a fraction of their maximum output during most of their operational life, so the distribution networks are underutilised most of the time [1]. To reduce electricity costs for consumers, the utilisation of the existing distribution assets should be maximised [1]. Active Network Management (ANM) techniques operate the network closer to its constraints by real time monitoring and control of network parameters, such as currents, voltages, DG outputs and responsive or nonresponsive load demands. Thus their utilisation will allow more renewable power resources to be connected to the existing distribution networks by maximising the utilisation of network assets [2].

EV car parks, which are also connected to the distribution level, can participate in such ANM schemes. It is possible to take advantage of the broad complementarity between the daily population profile of EVs in such car parks and the generation output profile of nearby solar farms by designing a control strategy to charge these EVs to maintain the grid parameters within their acceptable constraints. In this way, the controlled variable demand of EV car parks can help to increase the integrated solar power capacity within the network.

Non-optimal connection of EVs within the power system can potentially affect the quality of energy supply and damage power system equipment. In addition, it can also result in violation of the power system constraints, so an optimal integration of EVs in the network should be implemented to make sure that they can improve the power system operation. The aim of Optimal Power Flow (OPF), which is a technique for optimal operation and planning of power systems [3], is to optimise objective functions such as the amount of losses on the power system by setting some control variables in an optimal way while satisfying load demand and grid operating constraints [3]. The extended OPF formulation is a modified version of the standard OPF formulation, which includes additional variables, costs and/or equality and inequality constraints [4].

In this work, a novel extended OPF algorithm for control of EVs in car parks operating within radial distribution networks is proposed and its performance is assessed using a simulator developed within the MATLAB environment. The objective of the control strategy is to increase the capacity of solar power generation within the network while avoiding the cost of grid upgrade. The control strategy considers the EV capacity, 
Battery State of Charge (SoC), EV movements and the requirement of $\mathrm{EV}$ users and power system operator.

\section{Methodology}

In this work, a number of EV car parks and solar farms are added to a feeder of a radial distribution network. The controller tries to inject some of the surplus power from these solar farms to the EV car parks. The following steps are proposed to control these EVs within a feeder of a radial distribution network so as to maximise the utilisation of grid assets while respecting the power system constraints. The aim is to increase the local solar penetration whilst charging the EVs with green energy.

1) A number of solar farms will be added to a feeder of a radial distribution network without any storage until they breach the power system constraints during the simulation period or require curtailment to meet the constraints.

2) A number of EV car parks will be added to the same feeder of the network. They will have a reasonable distance from each other and will not be placed on the same buses as solar farms in order to reflect locational constraints. It is assumed that all car parks will have the same total EV capacity. The aggregate rating of filling stations will be chosen to be close to the aggregate maximum solar power generation during the simulation to make sure that the car parks are able to accept the maximum surplus of the feeder.

3) An extended Optimal Power Flow (OPF) controller with a cost function will be used to maximise the electricity demand of the EV car parks and minimise the distribution losses at each time step while satisfying the power system constraints and respecting the requirements of EV users. The reason to maximise the demand of each station is to increase the amount of electricity being sold to EVs through car parks and consequently maximise the profit of car park owners. The electricity demand of each car park will be determined from the optimisation results.

Figure 1 shows the algorithm used at each time interval of the simulation to find the charging demand of EVs within the network.

\section{Modelling details}

The United Kingdom Generic Distribution System (UKGDS) is a resource for simulation and analysis of the impact of distributed generation on the UK power network. The models represent the most common architectures used by the UK Distribution Network Operators (DNOs), but they are slightly altered to facilitate testing and evaluation of new concepts [5]. As shown in Figure 2, a High Voltage $(H V)$ Underground $(U G)$ $U K G D S$ is used as a case study in this work. A MATLAB GUI simulator, which uses the MATPOWER power flow engine [4], is developed to assess the performance of the proposed control strategy.

The electricity demand profile for the United Kingdom [6] is scaled down to the level acceptable by the UKGDS system, and then it is used in the simulation process to represent the nonEV demand on each node of the network. The share of the non-
EV loads on each bus is equal to the proportion of loads defined in the $U K G D S$ standard load profile [6]

In this work, the EV car parks and solar farms are modelled on only one feeder of the system (feeder number 8 , which is the last one) to assess the performance of the proposed control strategy. Table 1 shows the details of car parks and solar farms. The car parks are added on three buses, and the solar farms are also added at bus 58 and 63 of the UKGDS model. To scale the solar farms to the $U K G D S$ model and cause a violation of power system constraints without utilisation of EVs, their nominal generation capacity was selected to be $35 \mathrm{MW}$.

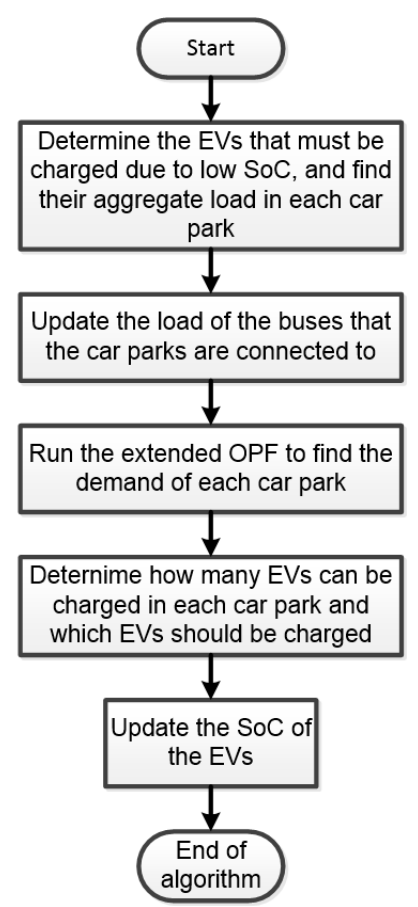

Figure 1: The algorithm used at each time interval to control the demand of car parks

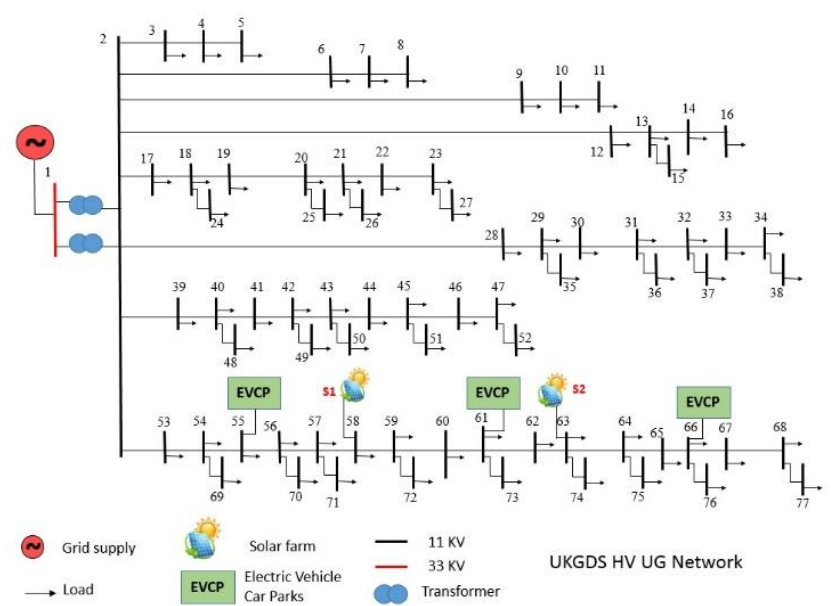

Figure 2: UKGDS HV UG network with solar farms and EV car parks

To simulate the solar power generation, the power output data from two solar sites in the UK was obtained from Microgen 
Database provided by Sheffield Solar group located at the University of Sheffield [7]. The output power from each site was scaled up to represent the solar power generation capacity in Table 1.

\begin{tabular}{|l|l|l|l|}
\hline Name & Bus number & $\begin{array}{l}\text { Nominal } \\
\text { Number of } \\
\text { EVs }\end{array}$ & $\begin{array}{l}\text { Rating } \\
(\text { MW) }\end{array}$ \\
\hline Solar farm 1 & 58 & - & 35 \\
Solar farm 2 & 63 & - & 35 \\
Car park 1 & 55 & 2000 EVs & 6 \\
Car park 2 & 61 & 2000 EVs & 6 \\
Car park 3 & 66 & 2000 EVs & 6 \\
\hline
\end{tabular}

Table 1: Details of car parks and solar farms

To carry out the analysis for the system under investigation, the following assumptions were made by the authors. It is assumed that each car park can accommodate $2000 \mathrm{EV}$, and each EV will be charged with a $3 \mathrm{~kW}$ charger, so the total power capacity of each car park is 6MW. The nominal capacities of all EVs are assumed to be equal at a capacity selected by the simulator user in units of $\mathrm{kWh}$.

All of the parked EVs are assumed to be connected to the grid and are able to be instructed by the control centre to be charged if required. This means that there exists a robust and reliable communication system between the Distribution Network Operator (DNO) control centre and each parked vehicle. This system sends some information about each EV to the control centre and receives an instruction from the control centre to adjust the demand of each EV within the system.

It is assumed that the EVs inside car parks belong to people who are working in nearby commercial or business centres. The simulator allocates the same travel pattern with the same timings to each EV for all simulation days, but different vehicles have different start and duration times for their daily journey. It is assumed that EVs move into the car parks in the morning at a random but constant time between $7 \mathrm{am}$ to $11 \mathrm{am}$ every day, and then they leave the car parks at a random time between $4 \mathrm{pm}$ to $6 \mathrm{pm}$ with 10 minute resolution. The travel duration of each EV is a random time between 10 minutes to 1.5 hours with a resolution of 10 minutes.

The simulator does not change the SoC of the EV battery when the $\mathrm{EV}$ is parked and not instructed by the control centre to be charged. The simulator user can input a minimum and maximum SoC limit for the EV batteries. When each car gets to a car park, the software allocates a random initial SoC between the minimum and maximum SoC limits to each battery. The minimum SoC limit selected by the user is the absolute minimum value which should not be reached even at the end of a journey, and it is the same for all of the EVs within the system. Therefore, the simulator has to find another secondary minimum SoC limit for each EV based on the amount of energy it consumes during its next journey to make sure that the EV batteries do not reach this limit. In the control strategy, the simulator uses this secondary limit which is not necessarily the same value for all EVs.

The vehicles are assumed to have constant weight, speed and route in their journeys, so the energy (kWh) spent by each EV in its journey back home are assumed to be the same each day.
To find this energy consumption the simulator assumes that each EV travels with the average speed of 20 miles/h [8] during a commute, and on average it consumes $0.25 \mathrm{kWh}$ energy per mile it travels [9]. All of batteries in an EV are modelled with one battery in this work. Therefore, when an EV is moving the SoC of its battery reduces according to the following equation after each simulation time step:

$\operatorname{SoC}_{E V_{i}}^{k+1}=S o C_{E V_{i}}^{k}-\frac{\bar{v} \times \bar{E}}{C} \times \delta \times 100$

where

' $k$ ' is the time interval number in the simulation (each one represents 10 minutes)

$S o C_{E V_{i}}^{k}$ is the SoC of the battery of the $i^{t h} \mathrm{EV}$ in percentage at the time interval $k$.

$\bar{E}$ is the average energy consumed by each vehicle in $\mathrm{kWh} / \mathrm{mile}$.

$\bar{v}$ is the average speed of each vehicle in mile/hour.

' $\mathrm{C}$ ' is the capacity of the battery in each $\mathrm{EV}$ in $\mathrm{kWh}$.

$\delta$ is the time interval of the simulation which is $1 / 6$ of an hour. In addition, when an EV is parked and being charged, the simulator uses the following equation to calculate the change in the SoC of its battery after each time step:

$S_{o} C_{E V_{i}}^{k+1}=S o C_{E V_{i}}^{k}+\frac{R}{C} \times \delta \times 100$

where

' $R$ ' is the rate of charge of the EVs in $\mathrm{kW}$.

Table 2 shows some inputs entered into the simulator before starting the simulation.

\begin{tabular}{|l|l|}
\hline Name & Value \\
\hline Nominal capacity of each EV (C) & $60(\mathrm{kWh})$ \\
Minimum SoC limit (Min_SoC) of each EV & $20 \%$ \\
Maximum SoC limit (Max_SoC) of each EV & $95 \%$ \\
\hline
\end{tabular}

Table 2: Simulator inputs

Two scenarios are considered in the simulations. In the first scenario, the system only has two solar farms without any EV car parks, and the fluctuation in the difference between the local generation and demand must as far as possible be compensated by import/export of power from the distribution substation. In the second scenario, EV car parks are also operating in the system to capture some of the surplus solar power generated within the feeder to alleviate the problems caused by the distributed solar generation within the network. A cost function $(\operatorname{Cost}(\mathrm{k}))$ is defined to maximise the electricity demand from car parks while minimising the losses within the distribution system. The objective of the optimisation is to find the optimal demand of each station to minimise Cost $(\mathfrak{E})$ at each simulation time step.

$\operatorname{Cost}(k)=C_{1} * \delta * \sum_{i=1}^{N B} P_{\text {ooss }_{i}}^{k}-C_{2} * \delta * \sum_{i=1}^{N C} C D_{i}^{k}$

where

$C_{i}$ are the cost function coefficients.

$N B$ is the number of branches on the power system.

$P_{\text {Loss }_{i}}^{k}$ is the amount of power loss on branch ' $\mathrm{i}$ ' of the power system at the time interval ' $k$ ' in MW.

$N C$ is the number of car parks on the feeder.

$C D_{i}^{k}$ is the demand from car park ' $i$ ' during the current time interval of ' $k$ ' in MW. 
$C_{1}$ is the cost of electricity loss and selected to be $£ 35 / \mathrm{MWh}$ [10], and $C_{2}$ is the price of electricity sold to EVs via car parks and is equal to $70 £ / \mathrm{MWh}$.

It is assumed that the EVs, which have low SoC and are not able to finish their next journey without being charged, will be charged immediately when they reach a car park irrespective of the result of the optimisation process. This type of charging is referred to as 'compulsory charging' in this work. After running the extended optimal power flow the demand of each car park will be known, and the number of cars that can be charged, in addition to the above mandatory charging ones, will be determined. The EVs with lower SoC have priority for being charged at each car park.

There are some limits on the demand of car parks and also power system constraints that should be respected during the optimisation process. Before detailing those limits, some additional variables are defined here.

The surplus solar power on the last feeder of the network can be calculated from the following equation. The controller needs to know the amount of solar generation and non-EV demand on each bus of the feeder at each time step in order to calculate the surplus solar generation.

$\operatorname{Surplus}(\mathrm{k})=\sum_{i=1}^{N S} \operatorname{Solar}_{i}^{k}-\sum_{i=53}^{77} D_{i}^{k}$

where

Solar $_{i}^{k}$ is the output of solar farm ' $i$ ' in MW at the current time step $k$.

$N S$ is the number of solar farms on the considered feeder.

$D_{i}^{k}$ is the amount of demand (excluding the demand of EVs) in MW on bus ' $i$ ' of the last feeder (from bus 53 to bus 77) at the current time step $k$.

The 'Surplus' value could become negative at some points when the aggregate solar power generation is below the aggregate local non-EV demand. In addition, there is a possibility that the aggregate demand from the EVs, which need compulsory charging might become higher than the surplus power on the feeder. Therefore, another variable called 'Aggregate Non Compulsory Car park Demand Limit' $(A N C C D L)$ is defined to be used as the limit in the simulations to make sure the that the EVs, which do not need compulsory charging, will be changed in the case that there is surplus solar power left on the feeder after satisfying non-EV demand and the demand of EVs that require compulsory charging. In other words, the EVs which do not need compulsory charging will only get charged with renewable power, and when the 'Surplus' value is negative, only the EVs which need compulsory charging will be charged. This decision is made to ensure that EVs will be charged with renewable power available on the system rather than the power from conventional power plants, which result in the production of significant amount of carbon dioxide.

$\operatorname{ANCCDL}(\mathrm{k})=\max \left(\operatorname{Surplus}(k)-\sum_{i=1}^{N C} C C D_{i}^{k}, 0\right)$

where

$C C D_{i}^{k}$ is the compulsory charging demand from car park ' $\mathrm{i}$ ' during the current time interval of ' $k$ ' in MW.

$A N C C D L$ will always have a non-negative value. This means that if there is no surplus power left on the feeder after satisfying compulsory charging, then $\operatorname{ANCCDL}(\mathrm{k})$ will be equal to zero.
The limits for the aggregate demand of the car parks are defined by the following equation.

$0 \leq \sum_{i=1}^{N C}\left(C D_{i}^{k}-C C D_{i}^{k}\right) \leq A N C C D L(\mathrm{k})$

In the cases where the aggregate surplus power becomes negative or zero, the demand of the EVs (excluding the ones which must be charged) will be zero to avoid the case of charging EVs with non-renewable power. In such cases, some limited power will also be imported from the substation to supply some of the local non-EV or compulsory EV charging demands which were not fully supplied due to lack of local solar power generation.

The thermal and voltage constraints of the power system are also applied in the optimisation control strategy.

\section{Simulation results and discussions}

In this section, the results of the simulated scenarios for a duration of 24 hours are presented and discussed.

Figure 3 shows the demand from the three EV car parks within the network during the simulation. The result show that the energy delivered to car park 2 , which is located at bus 61 , is higher than the one delivered to other stations. This means that the location of this particular car park makes it more suitable to help alleviate the problems created on the network as a result of adding solar farms to the feeder.

In addition, by applying the proposed control strategy, different amount of energy is delivered to different car parks, which means that the income from individual car parks will not be the same.

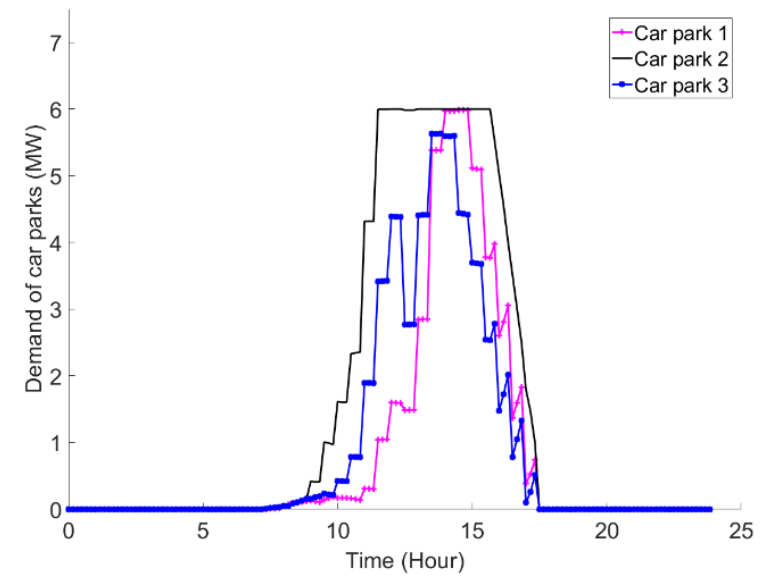

Figure 3: Electric demand of car parks during the simulation

Figure 4 shows the aggregate surplus solar power on feeder 8 and also the aggregate demand from all car parks. As specified in the control strategy in inequality (6), the aggregate demand of car parks should stay below the surplus solar power within the system unless the demand for compulsory charging of some EVs is above the surplus power on the feeder. The total amount of solar energy absorbed by the network during one day was equal to 104.1 MWh, and about 77.9 MWh of energy was used by EVs in car parks.

With the introduction of the EVs to the system, the voltages on different system nodes change. For example, the voltage on bus 63 , which has a nominal voltage of $11 \mathrm{KV}$, is shown in Figure 
5. This bus was selected because it had the maximum voltage rise as a result of adding solar farms without the utilisation of EV car parks. After utilisation of smart EV charging system, the voltage of the bus remained within the acceptable limits.

The simulation results show that the voltage limit on many buses were breached at least once during the simulation in the system without EVs, and that all of them are driven back within the limits as the result of utilisation of the control strategy with EV car parks.

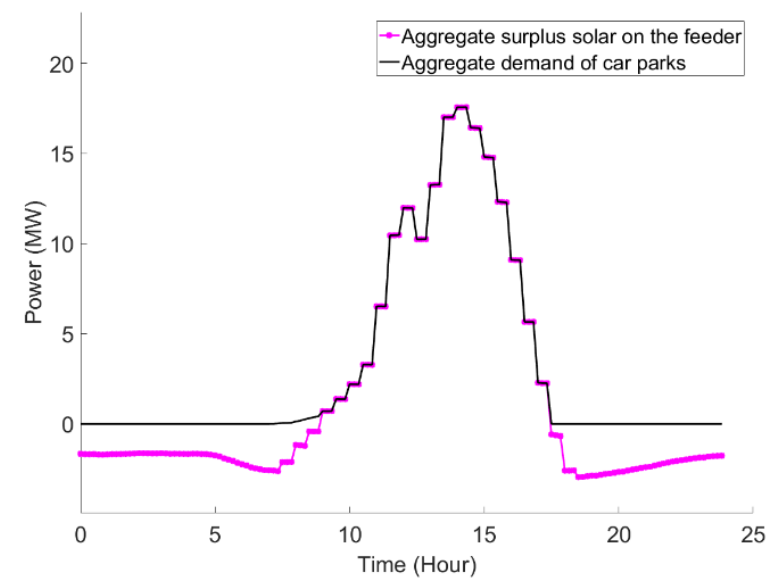

Figure 4: Aggregate surplus solar power and aggregate demand of car parks

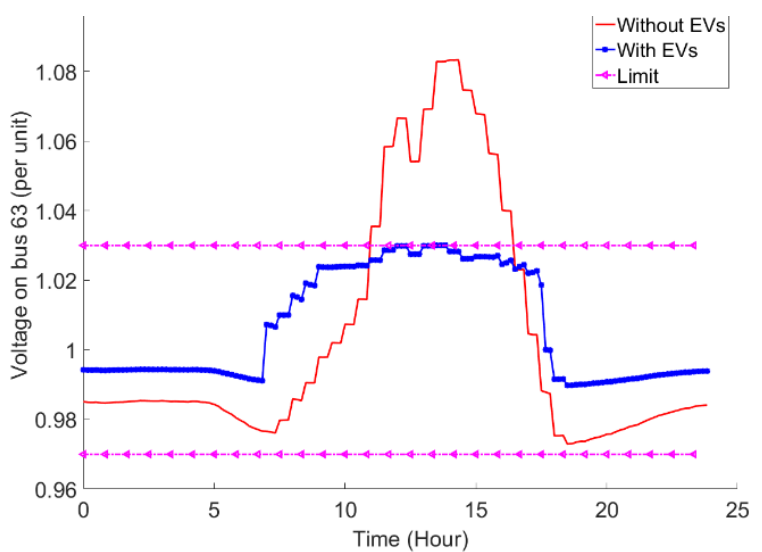

Figure 5: The voltage on bus 63 before and after adding EV car parks to the system

Figure 6 shows the amount of apparent power on the branch of power system which has the maximum peak value, in percentage terms, without using EVs during the simulation. It is obvious that the after using the EV car parks within the system the apparent power of this branch was controlled to remain within the acceptable limits. The simulation results show that the apparent power limit on some branches (from 53 to 63) were breached at least once during the 24 hour simulation in the system with solar farms but without EVs, and all of them were driven back within the limits as the result of utilisation of the control strategy with EV car parks.

On the other hand, there were some branches of the power system which were underutilised in the system without EVs and their apparent power peak was only a fraction of the nominal capacity limit of the branch. Figure 7 shows the apparent power of branch 64 with and without utilisation of EV car parks within the network. It has reached a much higher average apparent power while operating with EV car parks. The one day simulation results show that the probability of voltage constraint violation and thermal limit violations [11] were $22.9 \%$ and $20.8 \%$, respectively, before adding EV car parks to the power system. However, after smart charging of EVs, those values were found to be zero due to successful enforcement of the constraint limits by the system central controller. This shows the effectiveness of the control strategy to increase the utilisation of network assets and to remove the need for grid upgrades and associated costs while respecting the power system constraints and charging EVs with carbon free electricity.

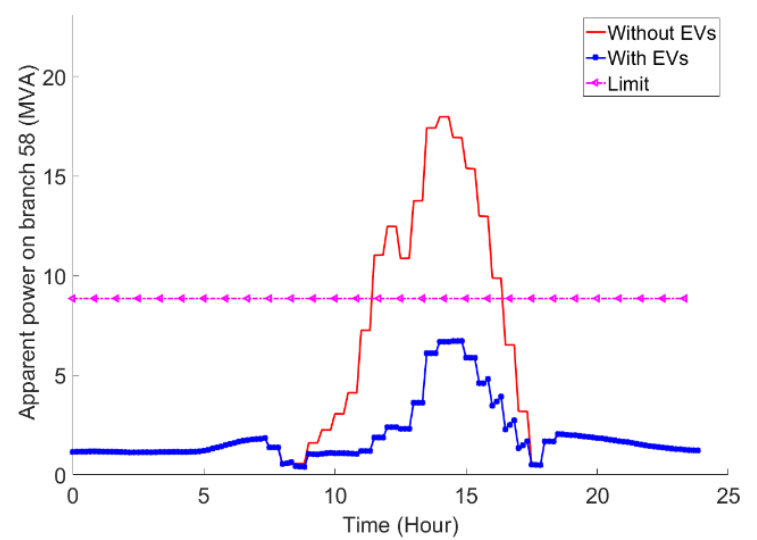

Figure 6: Apparent power on a branch of power system with the biggest peak percentage during the simulation

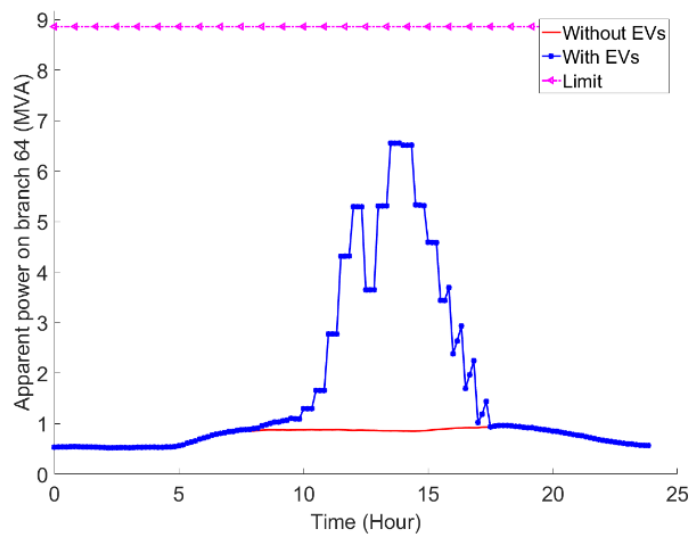

Figure 7: Apparent power on an underutilised branch of the power system during the simulation

The energy flow from the network to the EV car parks caused a reduction of $4.1 \mathrm{MWh}$ in the total energy loss of the distribution network. This is around $72.2 \%$ less than the distribution loss on the system without EVs. Despite the fact that the EVs act as additional demand on the electrical network, they reduced the distribution losses significantly in this study. The reduction in distribution losses is due to consumption of power generated by solar farms with local EV demand rather than exporting all of the surplus power to the other feeders. 
One of the advantages of the presented control strategy used in this work is that there is no need to forecast the solar power availability within the system, and it is assumed that the grid control centre can just use the real-time data from the solar power generation units, the local non-EV demand and each EV requirement and $\mathrm{SoC}$ to calculate the set-point for the demand of each car park.

Obviously, the system operator cannot add unlimited capacity of solar farms and EV demand to the system expecting that the controller should achieve the power system constraint limits. If more solar farms were added to the system, then they would generate more power, and more EVs could be added to the network to absorb this extra energy. However, the power system operator should make sure that the network limits will not be violated as a result of adding extra solar power capacity or EV demand in the scenarios using demand side management techniques.

Such smart charging techniques can help in increasing the amount of renewable power generation within the power system and consequently decrease carbon dioxide emission in electricity sector while avoiding the costs of grid upgrade. The system operator might also offer some incentives such as electricity cost reduction to the EV car park owners to participate in such schemes, which could eventually lead to a decrease in cost of $\mathrm{EV}$ charging, further encourage their adoption.

\section{Conclusions}

In this paper, a novel approach that uses an extended OPF was proposed to control the demand of EV car parks to increase the amount of solar power generation capacity within a radial distribution network while satisfying the power system constraints and EV user requirements. The effectiveness of the strategy has been proved through formulation of the problem and then simulation in MATLAB using a $U K G D S$ case study. The control strategy was able to increase the network asset utilisation while considering the EV user requirements and completely satisfying the voltage and thermal limit constraints during the simulation. The energy flow from the network to the EVs caused a significant reduction in the total energy loss of the distribution network during the simulation. Despite the fact that the EVs act as additional demand on the electrical network, they reduced the distribution losses significantly in this study. The reduction in distribution losses is due to the consumption of some of the surplus power generated by solar farms by the EVs on the local feeder, instead of exporting all of the surplus power to other parts of the network.

\section{Acknowledgements}

This work is a part of the Battery Characterisation and Management (BaChMan) project funded by the Engineering and Physical Sciences Research Council (EPSRC) in the UK and National Natural Science Foundation of China (NSFC) (grant reference: EP/L001004/1).

\section{References}

[1] J. A. P. Lopes, N. Hatziargyriou, J. Mutale, P. Djapic, and N. Jenkins, "Integrating distributed generation into electric power systems: A review of drivers, challenges and opportunities", Electric Power Systems Research, volume 77, no. 9, pp. 1189-1203, (2007).

[2] S. N. Liew, and G. Strbac, "Maximising penetration of wind generation in existing distribution networks", IEE Proceedings in Generation, Transmission and Distribution, volume 149, no. 3, pp. 256-262, (2002).

[3] P. D. Bamane, A. N. Kshirsagar, S. Raj, and H. Jadhav, "Temperature dependent Optimal Power Flow using gbest-guided artificial bee colony algorithm", in International Conference on Computation of Power, Energy, Information and Communication (ICCPEIC 2014), pp. 321-327, (2014).

[4] R. D. Zimmerman, C. E. Murillo-Sanchez, and R. J. Thomas, "MATPOWER's extensible optimal power flow architecture", in Power \& Energy Society General Meeting, 2009 (IEEE PES 09), pp. 1-7, (2009).

[5] C. Foote, P. Djapic, G. Ault, J. Mutale, and G. Strbac, "United Kingdom Generic Distribution System (UKGDS), Defining the Generic Networks", DTI Centre for Distributed Generation and Sustainable Electrical Energy, (2005).

[6] National grid website, "Metered half-hourly electricity demands", http://www.nationalgrid.com/uk/Electricity/Data/Deman d+Data/ (2015).

[7] Sheffield Solar, University of Sheffield, "Microgen Database ", http://www.solar.sheffield.ac.uk/panel-data/, (2015).

[8] "National Travel Survey Scottish Results during 20072008", The Scottish Government website, (2010).

[9] Tesla Motors website, http://www.teslamotors.com/, (2014).

[10] The Balancing Mechanism Reporting System (BMRS) website, "Historic System Prices", http://www.bmreports.com/bsp/SystemPricesHistoric.ht m, (2014).

[11] A. D. Alarcon-Rodriguez, "A Multi-objective Planning Framework for Analysing the Integration of Distributed Energy Resources", PhD thesis, Department of Electronic and Electrical Engineering, University of Strathclyde, (2009). 